\title{
Parameters and Fractional Differentiation Orders Estimation for Linear Continuous-Time Non-Commensurate Fractional Order Systems
}

\author{
Zehor Belkhatir, ${ }^{\mathrm{a}, *}$, Taous Meriem Laleg-Kirati ${ }^{\mathrm{a}}$ \\ ${ }^{a}$ Divison of Computer, Electrical and Mathematical Sciences and Engineering (CEMSE), \\ King Abdullah University of Science and Technology (KAUST), Saudi Arabia
}

\begin{abstract}
This paper proposes a two-stage estimation algorithm to solve the problem of joint estimation of the parameters and the fractional differentiation orders of a linear continuous-time fractional system with non-commensurate orders. The proposed algorithm combines the modulating functions and the first-order Newton methods. Sufficient conditions ensuring the convergence of the method are provided. An error analysis in the discrete case is performed. Moreover, the method is extended to the joint estimation of smooth unknown input and fractional differentiation orders. The performance of the proposed approach is illustrated with different numerical examples. Furthermore, a potential application of the algorithm is proposed which consists in the estimation of the differentiation orders of a fractional neurovascular model along with the neural activity considered as input for this model.
\end{abstract}

Key words: Linear fractional order systems, non-commensurate orders, parameters and fractional differentiation orders estimation, modulating functions method.

\section{Introduction}

The concept of fractional calculus, a generalization of integerorder integration and differentiation operators, is ancient and goes back to the seventeenth century. Thanks to its interesting properties of non-locality and memory, fractional derivative has been used for modeling complex phenomena in different fields of science and engineering [1], [2], [3]. When fractional models are developed, it may often happen that differentiation orders, parameters and/or input of those models are unknown. So, they have to be estimated from the available measurements. However, solving parameters and unknown input estimation problem for fractional order systems (FOS) is not straightforward, and the problem becomes more challenging when information about the fractional differentiation orders is missing as well.

Several estimation methods for FOS have been proposed in the literature. For instance, a fractional version of the extended Kalman filter has been proposed in [4] to estimate the unknown parameters and the fractional differentiation orders of a discrete FOS; a fractional version of Zak-Walcott sliding mode observer is introduced in [5] for the joint estimation of the pseudo-states and the unknown input of FOS. Victor et al. [6] associated a simplified refined instrumental variable method with a GaussNewton approach to estimate the unknown parameters and the fractional differentiation orders. Other estimation techniques can be found in [7], [8], [9], [10], and references therein.

The modulating functions (MFs) method has been introduced in the fifties for parameters' estimation of integer-order

\footnotetext{
${ }^{*}$ Corresponding author

Email addresses: zehor.belkhatirekaust.edu.sa (Zehor Belkhatir,), taousmeriem. laleg@kaust.edu.sa (Taous Meriem Laleg-Kirati)
}

systems [11]. It has been extended to FOS in [12] where the fractional differentiation order has been reduced to an integer order in the noise-free case. In [13], it has been used to estimate the parameters of FOS with known fractional differentiation orders. Moreover, the MFs method has been combined with an iterative method to estimate both the coefficients and a single differentiation order of a fractional advection-dispersion equation [14].

In this paper, we propose a hybrid algorithm which combines MFs and first-order Newton methods to jointly estimate the fractional differentiation orders and the parameters of fractional ordinary differential equations (FODEs). The approach is also extended to solve the estimation problem of smooth unknown input simultaneously with fractional differentiation orders. Our contributions are as follows: (i) we consider an initialized definition of fractional derivative; (ii) we extend the hybrid algorithm proposed in [14] for the general class of noncommensurate FODEs; and (iii) we perform an error analysis in the discrete case. The proposed method has many advantages. It transfers the fractional differentiation orders from the output, which is often noisy, to the MFs whose fractional derivative can be analytically computed. It also helps in characterizing the Jacobian of Newton's method at each iteration which reduces the computational cost. A potential application of the method is the estimation of the differentiation orders of a fractional neurovascular model along with the neural activity considered as input to this model.

The remainder of the paper is organized as follows. Section 2 recalls definitions and concepts on fractional calculus and MFs. In section 3, the estimation problem is formulated. In section 4 , the developed algorithm is described. Section 5 presents sufficient conditions that ensure the convergence of 
the proposed two-stage algorithm. An error analysis in discrete case is performed in section 6 . Section 7 extends the proposed method to the case of unknown input and fractional differentiation orders estimation. The performance of the algorithm is shown in section 8 through different numerical examples. Concluding remarks and future work are given in section 9 .

\section{Preliminaries}

In this section, some definitions and properties of initialized Rieman-Liouville (RL) fractional derivative and MFs are presented.

\subsection{Fractional derivative}

Definition 1. [15] The initialized RL fractional derivative is defined as

$$
D_{t}^{\alpha} f(t)=d_{t}^{\alpha} f(t)+\Psi\left(f_{i},-p,-a, 0, t\right), \quad t>0,
$$

where $\alpha=n-p$ with $n \in \mathbb{N}$ and $p, \alpha \in \mathbb{R}_{+}^{*}$. The terms $d_{t}^{\alpha} f(t)$ are the uninitialized $\alpha^{\text {th }}$ order $R L$ derivative and $\Psi\left(f_{i},-p,-a, 0, t\right)$ is the initialization function given as follows

$$
\left\{\begin{array}{l}
d_{t}^{\alpha} f(t)=\frac{d^{n}}{d t^{n}} \frac{1}{\Gamma(p)} \int_{0}^{t}(t-\tau)^{p-1} f(\tau) d \tau \\
\Psi\left(f_{i},-p,-a, 0, t\right)=\frac{d^{n}}{d t^{n}} \frac{1}{\Gamma(p)} \int_{-a}^{0}(t-\tau)^{p-1} f_{i}(\tau) d \tau
\end{array}\right.
$$

where $\Gamma($.$) is the gamma function and f_{i}(t)$ is the initialization function defined for $t \in[-a, 0], a \in \mathbb{R}_{+}^{*}$. This definition assumes that the history-function for $t \in(-\infty,-a)$ is zero.

The following lemma provides an analytic closed form for the derivative of $D_{t}^{\alpha} f(t)$ with respect to $\alpha$.

Lemma 1. If the $\alpha^{\text {th }}$ initialized RL derivative of a function $f(t)$ given in (1) exists, then the derivative of $D_{t}^{\alpha} f(t)$ with respect to $\alpha$ is given by

$$
\begin{gathered}
\frac{\partial}{\partial \alpha}\left[D_{t}^{\alpha} f(t)\right]=\psi_{0}(p) D_{t}^{\alpha} f(t)-\frac{1}{\Gamma(p)} \frac{d^{n}}{d t^{n}}\left[\int_{0}^{t}(t-\tau)^{p-1} \ln (t-\tau)\right. \\
\left.f(\tau) d \tau+\int_{-a}^{0}(t-\tau)^{p-1} \ln (t-\tau) f_{i}(\tau) d \tau\right]
\end{gathered}
$$

where $\psi_{0}()=.\frac{\Gamma^{\prime}(.)}{\Gamma(.)}$ is the standard digamma function.

PROOF. The proof is completed by differentiating (1) with respect to $\alpha$.

\subsection{Modulating functions (MFs)}

Definition 2. [16] A function $\phi(t)$, defined on $[0, T]$, is a $M F$ of order $n$ if it satisfies the following properties

(P1) $\phi \in C^{n}([0, T])$;

$(P 2) \phi^{(i)}(0)=\phi^{(i)}(T)=0, i=0,1, \ldots, n-1$.
Based on [17], the MF $\phi(t)$ of order $n$ satisfies the following properties

(P3) $D_{t}^{\alpha} \phi(t)$ exists, $\quad \forall 0 \leqslant \alpha \leqslant n$;

(P4) $\left.D_{t}^{\alpha} \phi(t)\right|_{t=0}=0 ; \quad \forall 0 \leqslant \alpha \leqslant n$.

A generalization of an integration by parts-like formula proposed in [13] for uninitialized RL fractional derivative is given in the following lemma for the case of constant initialization function.

Lemma 2. Lets consider a function $f(t)$ and let $\phi(t)$ be a $M F$ of order $n$ with $n \in \mathbb{N}^{*}$. Assuming that the $\alpha^{\text {th }}$ initialized $R L$ fractional derivative of $f(t)$ exists, with $\alpha \in \mathbb{R}_{+}^{*}$ and the constant initialization function $f_{i}(t)=c$, initialized from $t=-a, a>$ 0 , then the following expression holds

$$
\begin{aligned}
& \int_{0}^{T} D_{t}^{\alpha} f(t) \phi(T-t) d t=\int_{0}^{T} f(T-t) D_{t}^{\alpha} \phi(t) d t \\
& \quad+\frac{c}{\Gamma(1-\alpha)} \int_{0}^{T} \phi(T-t)(t+a)^{-\alpha} d t-c D_{t}^{\alpha-1} \phi(T) .
\end{aligned}
$$

PROOF. The proof is provided in the Appendix.

\section{Problem statement}

Let us consider the following non-commensurate FOS

$$
y(t)+\sum_{i=1}^{N} a_{i} D_{t}^{\alpha_{i}} y(t)=u(t), \quad t \in[0, T]
$$

where $y(t) \in \mathbb{R}$ is the output, $u(t) \in \mathbb{R}$ is the input, $\left\{a_{i}\right\}_{i=1}^{N} \in \mathbb{R}^{*}$ are the unknown parameters and $\left\{\alpha_{i}\right\}_{i=1}^{N} \in\left\{\left(n_{i}-1, n_{i}\right)\right\}_{i=1}^{N}$, with $n_{i} \in \mathbb{N}^{*}$, are the unknown fractional differentiation orders assumed to be as follow: $0<\alpha_{1}<\alpha_{2}<\cdots<\alpha_{N}$; i.e. $n_{i}<n_{i+1}$ for $i=1,2, \ldots, N-1$.

We denote the vectors $\theta \in\left(\mathbb{R}^{*}\right)^{N}$ and $\alpha \in \mathscr{P}=\prod_{i=1}^{N}\left(n_{i}-1, n_{i}\right)$, $n_{i} \in \mathbb{N}^{*}$, as follows

$$
\theta=\left(\begin{array}{llll}
a_{1} & a_{2} & \cdots & a_{N}
\end{array}\right)^{t r}, \alpha=\left(\begin{array}{llll}
\alpha_{1} & \alpha_{2} & \cdots & \alpha_{N}
\end{array}\right)^{t r},
$$

where $(.)^{t r}$ denotes the transpose of a raw vector.

In this work, we are interested in solving the following estimation problem (EP1)

$(\mathrm{EP} 1)\left\{\begin{array}{l}\text { Given } y(t) \text { and } u(t), \text { for } t \in(0, T) \text {, find the unknown } \\ \text { fractional differentiation order vector } \alpha \text { jointly with the } \\ \text { unknown parameter vector } \theta .\end{array}\right.$

We will also show how to solve the estimation problem (EP2) given as follows

(EP2) $\left\{\begin{array}{l}\text { Given } y(t) \text {, for } t \in(0, T) \text {, and knowing the values of } \\ \left\{a_{i}\right\}_{i=1}^{N}, \text { find the unknown fractional order vector } \alpha \\ \text { jointly with the unknown input } u(t) .\end{array}\right.$

Remark 1. For the sake of clarity and simplification of the analysis, the method is presented on system (5). However, it can be extended to deal with a general class of FOS, where the fractional derivatives affect the input signal as well. 


\section{Main result}

In this section, we introduce a hybrid method which combines the MFs approach and the first-order Newton algorithm to solve (EP1). The proposed method is iterative and consists of two steps. While the first step solves the parameters' estimation problem at each iteration using MFs, the second one solves a nonlinear system of equations using Newton's method to find estimates for the fractional differentiation orders.

The main result is stated in proposition 1 .

Proposition 1. Consider the FOS (5) initialized with a constant history function $f_{i}(t)=c$, for $t \in[-a, 0], a>0$. Let $\left\{\phi_{m}(t)\right\}_{m=1}^{M+N}$ be a set of MFs. Then, at each iteration $k$, the parameters $\left\{a_{i}^{k}\right\}_{i=1}^{N}$ are estimated by the solution of the following linear system

$$
A^{k} \hat{\theta}^{k}=b,
$$

where $A^{k} \in \mathbb{R}^{M \times N}$, and

$$
\hat{\theta}^{k}=\left(\begin{array}{c}
\hat{a}_{1}^{k} \\
\hat{a}_{2}^{k} \\
\vdots \\
\hat{a}_{N}^{k}
\end{array}\right), b=\left(\begin{array}{c}
b_{1} \\
b_{2} \\
\vdots \\
b_{M}
\end{array}\right)
$$

with; for $m=1: M, j=1: N$,

$$
\left\{\begin{aligned}
A_{m j}^{k}= & {\left[\int_{0}^{T} D_{t}^{\alpha_{j}^{k}} \phi_{m}(t) y(T-t) d t-c D_{t}^{\alpha_{j}^{k}-1} \phi(T)\right.} \\
& \left.+\frac{c}{\Gamma\left(1-\alpha_{j}^{k}\right)} \int_{0}^{T} \phi_{m}(T-t)(t+a)^{-\alpha_{j}^{k}} d t\right], \\
b_{m}= & \int_{0}^{T} \phi_{m}(T-t)[u(t)-y(t)] d t,
\end{aligned}\right.
$$

and then the vector $\alpha$ is updated iteratively as follows

$$
\alpha^{k+1}=\alpha^{k}-\left[L^{\prime}\left(\alpha^{k}\right)\right]^{-1} J\left(\alpha^{k}\right)
$$

where $J\left(\alpha^{k}\right)$ is given by

$$
J\left(\alpha^{k}\right)=L\left(\alpha^{k}\right)-R=0,
$$

with $L\left(\alpha^{k}\right)$ and $R$ are vectors containing $N$ elements given, for $j=1: N$, as follows

$$
\left\{\begin{array}{l}
L_{j}\left(\alpha^{k}\right)=\sum_{i=1}^{N} a_{i}\left(\alpha^{k}\right) A_{(M+j) i}^{k} \\
R_{j}=\int_{0}^{T} \phi_{M+j}(T-t)[u(t)-y(t)] d t
\end{array}\right.
$$

and $L^{\prime}\left(\alpha^{k}\right) \in \mathbb{R}^{N \times N}$ is the Jacobian matrix given by

$$
L^{\prime}\left(\alpha^{k}\right)=\left(\begin{array}{cccc}
\frac{\partial L_{1}}{\partial \alpha_{1}}\left(\alpha^{k}\right) & \frac{\partial L_{1}}{\partial \alpha_{2}}\left(\alpha^{k}\right) & \cdots & \frac{\partial L_{1}}{\partial \alpha_{N}}\left(\alpha^{k}\right) \\
\vdots & \vdots & \vdots & \vdots \\
\frac{\partial L_{N}}{\partial \alpha_{1}}\left(\alpha^{k}\right) & \frac{\partial L_{N}}{\partial \alpha_{2}}\left(\alpha^{k}\right) & \cdots & \frac{\partial L_{N}}{\partial \alpha_{N}}\left(\alpha^{k}\right)
\end{array}\right)
$$

Proof. We multiply equation (5) by a set of MFs $\left\{\phi_{m}(T-t)\right\}_{m=1}^{M+N}$. Then, we integrate over $[0, T]$ to get, at each iteration $k$,

$$
\begin{aligned}
\int_{0}^{T} \phi_{m}(T-t) y(t) \mathrm{dt} & +\sum_{i=1}^{N} a_{i}^{k} \int_{0}^{T} \phi_{m}(T-t) D_{t}^{\alpha_{i}^{k}} y(t) \mathrm{dt} \\
& =\int_{0}^{T} \phi_{m}(T-t) u(t) \mathrm{dt} .
\end{aligned}
$$

We use formula (4) given in Lemma 2 to integrate equation (14), which yields to the following equation

$$
\sum_{i=1}^{N} a_{i}^{k} A_{m i}^{k}=\int_{0}^{T} \phi_{m}(T-t)[u(t)-y(t)] \mathrm{dt},
$$

where $A_{m i}^{k}$ are defined in equation (9).

Step 1: We consider the set of MFs $\left\{\phi_{m}(T-t)\right\}_{m=1}^{M}$. Thus, at each iteration $k$, equation (7) is obtained by rewriting equation (15) as a system of linear equations.

Step 2: First, let us write explicitly the dependence of the parameters on the fractional orders in (15); i.e. $\left\{a_{i}\left(\alpha^{k}\right)\right\}_{i=1}^{N}$;

$$
\sum_{i=1}^{N} a_{i}\left(\alpha^{k}\right) A_{m i}^{k}=\int_{0}^{T} \phi_{m}(T-t)[u(t)-y(t)] \mathrm{dt},
$$

Then, we consider the set of MFs $\left\{\phi_{m}(T-t)\right\}_{m=M+1}^{M+N}$ in (16). First-order Newton method allows us to write the update law given in equation (10). Equations (11) and (12) result from rearranging equation (16) as a nonlinear system of equations.

Thanks to the MFs, we can exactly characterize the entries of the Jacobian matrix as given in the following Lemma.

Lemma 3. The entries of the Jacobian matrix are analytically characterized as follows; for $\bar{i}=1: N, j=1: N$,

$$
\frac{\partial L_{j}}{\partial \alpha_{\bar{i}}}\left(\alpha^{k}\right)=\sum_{i=1}^{N} \frac{\partial a_{i}\left(\alpha^{k}\right)}{\partial \alpha_{\bar{i}}} A_{(M+j) i}^{k}+a_{\bar{i}}\left(\alpha^{k}\right) \frac{\partial A_{(M+j) \bar{i}}^{k}}{\partial \alpha_{\bar{i}}}
$$

The terms $\frac{\partial a_{j}\left(\alpha^{k}\right)}{\partial \alpha_{\bar{i}}}$ are estimated, at each iteration $k$, by the solution of the following linear system

$$
A^{k}\left(\hat{\theta}_{d}^{j}\right)^{k}=\left(\bar{b}^{j}\right)^{k}
$$

where the elements of $A^{k} \in \mathbb{R}^{M \times N}$ are given in (9), and

$$
\left(\hat{\theta}_{d}^{j}\right)^{k}=\left(\begin{array}{c}
\frac{\partial \hat{a}_{1}^{k}}{\partial \alpha_{j}} \\
\frac{\partial \hat{a}_{2}^{k}}{\partial \alpha_{j}} \\
\vdots \\
\frac{\partial \hat{a}_{N}^{k}}{\partial \alpha_{j}}
\end{array}\right), \bar{b}^{j}=\left(\begin{array}{c}
b_{1}^{j} \\
b_{2}^{j} \\
\vdots \\
b_{M}^{j}
\end{array}\right)
$$

with; for $m=1: M, j=1: N$,

$$
\left(\bar{b}_{m}^{j}\right)^{k}=-a_{j}^{k} \frac{\partial A_{m j}^{k}}{\partial \alpha_{j}}
$$


where

$$
\begin{aligned}
\frac{\partial A_{m j}^{k}}{\partial \alpha_{j}}= & \int_{0}^{T} \frac{\partial D_{t}^{\alpha_{j}^{k}} \phi_{m}(t)}{\partial \alpha_{j}^{k}} y(T-t) d t-c \frac{\partial D_{t}^{\alpha_{j}^{k}-1} \phi_{m}(T)}{\partial \alpha_{j}^{k}} \\
& +\frac{c}{\Gamma\left(1-\alpha_{j}^{k}\right)}\left[\Psi_{0}\left(1-\alpha_{j}^{k}\right) \int_{0}^{T} \phi_{m}(T-t)(t+a)^{-\alpha_{j}^{k}} d t\right. \\
& \left.-\alpha_{j}^{k} \int_{0}^{T} \phi_{m}(T-t)(t+a)^{-1-\alpha_{j}^{k}} d t\right] .
\end{aligned}
$$

The quantities $a_{m}\left(\alpha^{k}\right), \frac{\partial A_{(M+j) m}^{k}}{\partial \alpha_{m}}$, and $\frac{\partial D_{t}^{\alpha_{j}^{k}} \phi_{m}(t)}{\partial \alpha_{j}^{k}}$, are computed using (7), (21), and lemma 1 respectively.

Proof. The analytic closed form given in (17) is obtained by differentiating the variables $\left\{L_{j}\left(\alpha^{k}\right)\right\}_{j=1}^{N}$ of (12) with respect to $\left\{\alpha_{\bar{i}}\right\}_{\bar{i}=1}^{N}$. We take the partial derivatives of equation (16) with respect to $\alpha_{j}, j=1, \ldots, N$ and for $m=1: M$. Then, we rewrite the obtained equation as a system of linear equations to end up with (18). The proof is completed by differentiating the elements of the matrix $A^{k}$ given in equation (9) which yields to equation (21).

Remark 2. It is worth to note that, at each iteration, the total number of MFs that allow the estimation of both the parameters and the fractional differentiation orders is $M+N$. While in the first step we use $M M F s$, i.e. $m=1: M$, to estimate the parameters and their gradients with respect to $\alpha$, in the second one we use the following $N$ MFs, i.e. $m=M+1: M+N$, to estimate the fractional differentiation orders. This strategy has been adopted to avoid issues that may result from the dependence between the MFs.

The main Steps of the proposed algorithm are given in Table 1.

Step 1: Initialize $k=0$, give an initial guess to $\alpha^{0}$,

Step 2: Compute $\hat{\theta}^{k}$ using equation (7) of proposition 1,

Step 3: Compute $J\left(\alpha^{k}\right)$ using equations (11-12),

Step 4: • If $\left\|\frac{J\left(\alpha^{k}\right)}{R}\right\|_{2}<\varepsilon$, then stop,

- else update $\alpha^{k}$ using equations (10-13) and lemma 3, update the number of iterations $k=k+1$ and then return to step (2).

Table 1: Steps of the proposed two-stage algorithm

\section{Convergence of the algorithm}

The iterative character of the above algorithm results from the Newton method employed to estimate $\alpha$. Regarding the parameter vector $\theta$, an exact formula is derived to estimate it.
However, we note that the estimates of the parameters should be computed at each iteration since they depend on $\alpha$ (see equations 7-9).

Thus, we use a standard convergence result of Newton's method to provide sufficient conditions ensuring the convergence of the proposed two-stage algorithm. Such a result can be found in chapter 5 of [18] for example. We also make use of two results on Lipschitz functions stating that the product and quotient (denominator not zero) of two bounded Lipschitz functions are Lipschitz [19].

The following assumptions are needed to prove the convergence result stated in theorem 1 .

Assumption 1. The input $u(t)$ and output $y(t)$ are bounded functions in $[0, T]$.

Assumption 2. The functions $D_{t}^{\bar{\alpha}} \phi(t)$ and $\frac{\partial}{\partial \bar{\alpha}} D_{t}^{\bar{\alpha}} \phi(t)$ are Lipschitz continuous with respect to a fractional order $\bar{\alpha}$ belonging to the intervals of $\mathscr{P}$.

Assumption 3. A solution $\alpha^{*}$ to the system $J(\alpha)=0$ exists.

Assumption 4. The Jacobian matrix $L^{\prime}\left(\alpha^{*}\right)$ is nonsingular.

Theorem 1. Assume that assumptions (1-4) hold. Then the proposed MF-based method converges q-quadratically.

ProOF. Based on [18, Theorem 5.1.2, page 71], and given assumptions 3 and 4, it remains to prove that the Jacobian matrix is Lipschitz continuous for $\alpha \in \mathscr{P}$ in order to conclude about the convergence of the proposed algorithm.

The entries of the Jacobian matrix are given by equation (17) where the terms $a(\alpha)$ and $\frac{\partial a(\alpha)}{\partial \alpha_{j}}$ are characterized in proposition 1 and lemma 3.

The norm 1 of the term $L^{\prime}\left(\alpha^{1}\right)-L^{\prime}\left(\alpha^{2}\right)$ is given as follows

$$
\begin{aligned}
& \left\|L^{\prime}\left(\alpha^{1}\right)-L^{\prime}\left(\alpha^{2}\right)\right\|_{1}=\max _{1 \leq j \leq N} \sum_{i=1}^{N}\left|L_{i j}^{\prime}\left(\alpha^{1}\right)-L_{i j}^{\prime}\left(\alpha^{2}\right)\right| \\
& =\sum_{i=1}^{N} \mid \sum_{k=1}^{N}\left(\frac{\partial a_{k}\left(\alpha^{1}\right)}{\partial \alpha_{j^{*}}^{1}} A_{(M+i) k}\left(\alpha^{1}\right)-\frac{\partial a_{k}\left(\alpha^{2}\right)}{\partial \alpha_{j^{*}}^{2}} A_{(M+i) k}\left(\alpha^{2}\right)\right) \\
& \quad+\left(a_{j^{*}}\left(\alpha^{1}\right) \frac{\partial A_{(M+i) j^{*}}}{\partial \alpha_{j^{*}}}\left(\alpha^{1}\right)-a_{j^{*}}\left(\alpha^{2}\right) \frac{\partial A_{(M+i) j^{*}}}{\partial \alpha_{j^{*}}}\left(\alpha^{2}\right)\right) \mid,
\end{aligned}
$$

where $j^{*}$ is the column index corresponding to the maximum of the sum of the row components. Then, using triangle inequality, equation (22) can be written as follows

$$
\begin{aligned}
& \left\|L^{\prime}\left(\alpha^{1}\right)-L^{\prime}\left(\alpha^{2}\right)\right\|_{1} \leq N \sum_{k=1}^{N}\left|\frac{\partial a_{k}\left(\alpha^{1}\right)}{\partial \alpha_{j^{*}}^{1}} A_{\left(M+i^{*}\right) k}\left(\alpha^{1}\right)-\frac{\partial a_{k}\left(\alpha^{2}\right)}{\partial \alpha_{j^{*}}^{2}} A_{\left(M+i^{*}\right) k}\left(\alpha^{2}\right)\right| \\
& \quad+\left|a_{j^{*}}\left(\alpha^{1}\right) \frac{\partial A_{\left(M+i^{*}\right) j^{*}}}{\partial \alpha_{j^{*}}}\left(\alpha^{1}\right)-a_{j^{*}}\left(\alpha^{2}\right) \frac{\partial A_{\left(M+i^{*}\right) j^{*}}}{\partial \alpha_{j^{*}}}\left(\alpha^{2}\right)\right|
\end{aligned}
$$

where $i^{*}$ is the index corresponding to the maximum of the terms of the sum over $i$ given in equation (22).

The terms of the right hand side of inequality (23) are product of functions. Thus, to prove the lipschitz continuity of $L^{\prime}(\alpha)$ and using properties on Lipschitz functions [19] we have to 
prove that, for $m=1: M$ and $j=1: N$, the functions $A_{m j}(\alpha)$, $\frac{\partial A_{m j}}{\partial \alpha_{j}}, a_{j}(\alpha)$ and $\frac{\partial a_{k}(\alpha)}{\partial \alpha_{j}}$ are Lipschitz continuous for a fractional order laying in the intervals of $\mathscr{P}$. Thanks to the properties on Lipschitz functions [19] and based on the expressions of these latter functions given in (9), (21), (7), and (18), respectively, their Lipschitz continuity follows from the boundness of the vector $b$ given in (9) and the Lipschitz continuity of the following functions; $g_{1}(\alpha)=\int_{0}^{T} D_{t}^{\alpha_{j}} \phi(t) y(T-t) \mathrm{dt}$, $g_{2}(\alpha)=\int_{0}^{T} \frac{\partial}{\partial \alpha_{j}} D_{t}^{\alpha_{j}} \phi(t) y(T-t) \mathrm{dt}, g_{3}(\alpha)=\frac{1}{\Gamma\left(1-\alpha_{j}\right)}$, $g_{4}(\alpha)=\Psi_{0}\left(1-\alpha_{j}\right), g_{5}(\alpha)=\int_{0}^{T} \frac{\partial}{\partial \alpha_{j}} D_{t}^{\alpha_{j}} \phi(t)(t+a)^{-\alpha_{j}} \mathrm{dt}$ and $g_{6}(\alpha)=\alpha_{j} \int_{0}^{T} \frac{\partial}{\partial \alpha_{j}} D_{t}^{\alpha_{j}} \phi(t)(t+a)^{-\alpha_{j}} \mathrm{dt}$.

The boundness of the vector $b$ holds using assumptions 1 and the boundness of the MFs, which follows from the property (P1). The Lipschitz continuity of the functions $g_{1}(\alpha)$ and $g_{2}(\alpha)$ follows directly using assumptions 1 and 2 . The function $g(\alpha)=(t+a)^{-\alpha_{j}}$ is Lipschitz continuous for $t>-a$ which leads to the Lipschitz continuity of $g_{5}(\alpha)$ and $g_{6}(\alpha)$ using assumption 2. For the functions $g_{3}(\alpha)$ and $g_{4}(\alpha)$, properties on the gamma and digamma functions along with the reflection formulas [20] are used to conclude about their Lipschitz continuity.

Thus, we end up with the following

$$
\left\|L^{\prime}\left(\alpha^{1}\right)-L^{\prime}\left(\alpha^{2}\right)\right\|_{1} \leq \gamma\left\|\alpha^{1}-\alpha^{2}\right\|_{1}
$$

where the Lipschitz constant $\gamma$ depends on $N$, the bounds of $\phi(t), u(t)$ and $y(t)$ as well as the Lipschitz constants of $D_{t}^{\alpha} \phi(t)$, $\frac{\partial}{\partial \alpha} D_{t}^{\alpha} \phi(t), g_{i}(\alpha)$, gamma function and digamma function.

\section{Error analysis in discrete case}

In section 5, we developed the algorithm assuming that the output $y(t)$ is continuous and noiseless. However, in real applications, the output signal is generally discrete and corrupted by noise. In this section, we study the error contribution of the noise when the output is given by

$$
y_{\delta}\left(t_{i}\right)=y\left(t_{i}\right)+\delta\left(t_{i}\right),
$$

where $t_{i}=i T_{s}, i=0, \ldots, L$ are the equidistant sampling times separated with the sampling time $T_{s}=\frac{T}{L}, L \in \mathbb{N}^{*}$. The signals $y\left(t_{i}\right)$ and $y_{\delta}\left(t_{i}\right)$ are the noiseless and noisy discrete outputs. In addition, we consider a class of noises $\delta(t)$ that obeys the following conditions

(H1) $\delta\left(t_{1}\right)$ and $\delta\left(t_{2}\right)$ are independent, for any different $t_{1}$ and $t_{2}$ in $(0, T)$.

(H2) The mean value of $\delta(t)$, denoted by $\mathbb{E}[$.$] , is zero.$

(H3) The variance of $\delta(t)$, denoted by $\operatorname{Var}[$.$] , is bounded; i.e.$ $\operatorname{Var}[\delta(t)] \leq \sigma$
We point out that zero-mean white Gaussian noise satisfies these conditions.

We substitute the output $y(t)$ by the noisy one $y_{\delta}(t)$ in equations (7), (17), (18). Then, the noise will affect three types of continuous integrals appearing in matrix $A$, vectors $b, \bar{b}^{j}$ and matrix $L^{\prime}$. Since we have access to discrete measurements, these integrals are approximated by their discrete version; i.e. $\int_{0}^{T} f(t) \mathrm{dt} \approx T_{s} \sum_{i=0}^{L} w_{i} f\left(t_{i}\right)$, where $w_{i} i=1, \ldots, L$ are weights that depend on the numerical integration scheme. Therefore, the discrete version of these integrals is given by

$$
\left\{\begin{aligned}
I_{1}^{y^{\delta}, m} & =T_{s} \sum_{i=0}^{L} w_{i} \phi_{m}\left(t_{L-i}\right) y\left(t_{i}\right)+e_{1}^{m}, \\
I_{2}^{y^{\delta}, m} & =T_{s} \sum_{i=0}^{L} w_{i} D_{t}^{\alpha} \phi_{m}\left(t_{i}\right) y\left(t_{L-i}\right)+e_{2}^{m}, \\
I_{3}^{y^{\delta}, m} & =T_{s} \sum_{i=0}^{L} w_{i} \frac{\partial}{\partial \alpha} D_{t}^{\alpha} \phi_{m}\left(t_{i}\right) y\left(t_{L-i}\right)+e_{3}^{m},
\end{aligned}\right.
$$

where

$$
\left\{\begin{array}{l}
e_{1}^{m}=T_{s} \sum_{i=0}^{L} w_{i} \phi_{m}\left(t_{L-i}\right) \delta\left(t_{i}\right) \\
e_{2}^{m}=T_{s} \sum_{i=0}^{L} w_{i} D_{t}^{\alpha} \phi_{m}\left(t_{i}\right) \delta\left(t_{L-i}\right) \\
e_{3}^{m}=T_{s} \sum_{i=0}^{L} w_{i} \frac{\partial}{\partial \alpha} D_{t}^{\alpha} \phi_{m}\left(t_{i}\right) \delta\left(t_{L-i}\right)
\end{array}\right.
$$

Consequently, the proposed algorithm is corrupted by: (i) the numerical error contribution resulting from the numerical integration scheme, and (ii) the discrete noise errors contribution $e_{i}^{m}, i=1,2,3$. Regarding the numerical integration error, it is well-known that it can be reduced by decreasing the sampling time. However, for the noise error terms, we can show the following.

Proposition 2. Let $\delta(t)$ be the class of noises satisfying conditions (H1)-(H3). Given the properties of the MFs and lemma 1 along with the properties of gamma and digamma functions [20], then

$$
\mathbb{E}\left[e_{i}^{m}\right]=0, \quad i=1,2,3,
$$

and $\mathbb{E}\left[\left(e_{i}^{m}\right)^{2}\right]=\operatorname{Var}\left[e_{i}^{m}\right], i=1,2,3$ satisfy

$$
\left\{\begin{array}{l}
\mathbb{E}\left[\left(e_{1}^{m}\right)^{2}\right] \leq T_{s} \sigma\left(T_{s} \sum_{i=0}^{L} w_{i}^{2}\left[\phi_{m}\left(t_{L-i}\right)\right]^{2}\right) \underset{T_{s} \rightarrow 0}{\longrightarrow} 0, \\
\mathbb{E}\left[\left(e_{2}^{m}\right)^{2}\right] \leq T_{s} \sigma\left(T_{s} \sum_{i=0}^{L} w_{i}^{2}\left[D_{t}^{\alpha} \phi_{m}\left(t_{i}\right)\right]^{2}\right) \underset{T_{s} \rightarrow 0}{\longrightarrow} 0, \\
\mathbb{E}\left[\left(e_{3}^{m}\right)^{2}\right] \leq T_{s} \sigma\left(T_{s} \sum_{i=0}^{L} w_{i}^{2}\left[\frac{\partial}{\partial \alpha} D_{t}^{\alpha} \phi_{m}\left(t_{i}\right)\right]^{2}\right) \underset{T_{s} \rightarrow 0}{\longrightarrow} 0 .
\end{array}\right.
$$

ProOF. The technique of the proof follows the one given in [21]. However, we point out that, in addition to the properties of MFs stated in section 2, we make use of the properties of gamma and digamma functions [20]. 
Remark 3. The above result means that the discrete noise errors are zero-mean and their variances can be reduced as the sampling time goes to zero. However, if we assume that the output signal is given to us with a particular sampling time and noise statistical properties and the integration scheme is fixed, we can minimize the variance of noise errors contribution by the choice of the type of MFs.

\section{Extension of the proposed algorithm to joint estimation of the unknown input and the fractional differentiation orders}

This section extends the proposed method to solve (EP2) for smooth input functions. The main idea of solving that problem is to project the unknown input in an appropriate set of basis functions. As a consequence, the initial input inverse problem is transformed into an equivalent parameters inverse problem. This means that the algorithm which is developed to solve (EP1) can be adjusted to solve (EP2).

Let us decompose the input signal in the space spanned by a set of basis functions $\left\{\beta_{i}(t)\right\}_{i=1}^{V}$ as follows

$$
u(t)=\sum_{i=1}^{V} x_{i} \beta_{i}(t)
$$

where $\left\{x_{i}\right\}_{i=1}^{V}, V \in \mathbb{N}^{*}$, are the unknown projection parameters that will be estimated jointly with the vector of fractional orders $\alpha$. Following this projection, we propose to solve (EP2*) instead of (EP2).

$\left(\mathrm{EP} 2^{*}\right)\left\{\begin{array}{l}\text { Given } y(t), \text { for } t \in(0, T), \text { and knowing the values } \\ \text { of }\left\{a_{i}\right\}_{i=1}^{N}, \text { find the unknown parameter vector } \alpha \\ \text { jointly with the unknown projection weights }\left\{x_{i}\right\}_{i=1}^{V}\end{array}\right.$

In what follows, we show how the proposed algorithm can be modified to solve (EP2*).

1. System of linear equations (7) in proposition 1 is replaced by

$$
\tilde{A} \hat{x}^{k}=\tilde{b}^{k},
$$

where for $m=1: M, j=1: V$,

$$
\left\{\begin{array}{l}
\tilde{A}_{m j}=\int_{0}^{T} \phi_{m}(T-t) \beta_{j}(t) \mathrm{dt}, \\
\tilde{b}_{m}^{k}=\int_{0}^{T} \phi_{m}(T-t) y(t) \mathrm{dt}+\sum_{i=1}^{N} a_{i} A_{m i}^{k},
\end{array}\right.
$$

where $A_{m i}^{k}$ is given in (9).

2. Equation (12) becomes in this case, $\forall j=1: N$,

$$
\left\{\begin{array}{c}
\tilde{L}_{j}\left(\alpha^{k}\right)=\sum_{i=1}^{V} x_{i}\left(\alpha^{k}\right) \int_{0}^{T} \phi_{M+j}(T-t) \beta_{i}(t) \mathrm{dt} \\
-\sum_{i=1}^{N} a_{i} A_{(M+j) i}^{k}, \\
\tilde{R}_{j}=\int_{0}^{T} \phi_{M+j}(T-t) y(t) \mathrm{dt},
\end{array}\right.
$$

where $A_{(M+j) i}^{k}$ is given in (9).
3. The entries of the Jacobian matrix (17) are amended to be, $\forall(j, \bar{i})=1: N$,

$$
\begin{array}{r}
\frac{\partial \tilde{L}_{j}}{\partial \alpha_{\bar{i}}}\left(\alpha^{k}\right)=\sum_{i=1}^{V} \frac{\partial x_{i}\left(\alpha^{k}\right)}{\partial \alpha_{\bar{i}}} \int_{0}^{T} \phi_{M+j}(t) \beta_{i}(t) \mathrm{dt} \\
-a_{\bar{i}} \frac{\partial A_{(M+j) \bar{i}}^{k}}{\partial \alpha_{\bar{i}}} ;
\end{array}
$$

with $\frac{\partial A_{(M+j) \bar{i}}^{k}}{\partial \alpha_{\bar{i}}}$ given in (21).

4. System of equations (18) in lemma 3 is changed to

$$
\tilde{A}\left(\hat{x}_{d}^{j}\right)^{k}=\left(\tilde{b}^{j}\right)^{k}
$$

where, $\forall(j=1: N, i=1: V, m=1: M)$,

$$
\left\{\begin{array}{l}
\tilde{A}_{m i} \text { given in }(32), \\
\left(\tilde{b}_{m}^{j}\right)^{k}=-a_{j}^{k} \frac{\partial A_{m j}^{k}}{\partial \alpha_{j}} \\
\left(\hat{x}_{d}^{j}\right)_{i}^{k}=\frac{\partial \hat{x}_{i}^{k}}{\partial \alpha_{j}^{k}}
\end{array}\right.
$$

where $\frac{\partial A_{i j}}{\partial \alpha_{j}}$ is given in (21) and $\left(\hat{x}_{d}^{j}\right)_{i}^{k}$ denotes the $i^{t h}$ element of $\hat{x}_{d}^{j}$ at iteration $k$.

Given the changes defined above, the same algorithm described in Table I is used to estimate $\left\{x_{i}\right\}_{i=1}^{V}$ and thus $u(t)$, using equation (30), jointly with the differentiation order vector $\alpha$.

\section{Numerical results}

In order to assess the performance of the proposed twostage algorithm, two sets of numerical examples are considered. While in the first example, we estimate parameters and fractional differentiation orders, in the second one we estimate jointly smooth unknown input and fractional orders.

In all the simulations given below, we assume that the initialization function $f_{i}(t)=0, \forall t \in(-\infty, 0]$. We also use GrünwaldLetnikov scheme (37), which is shown to be equivalent to RL scheme in [17, chapter 2], to implement numerically the fractional derivatives.

$$
D_{t}^{\alpha} f(t)=\lim _{h \rightarrow 0} \frac{1}{h^{\alpha}} \sum_{i=0}^{[t / h]} c_{i}^{(\alpha)} f(t-i h), \quad \alpha>0,
$$

where $h>0$ is the time step, $c_{i}^{(\alpha)}(i=0,1, \ldots)$ are the binomial coefficients recursively computed using the following formula

$$
c_{0}^{(\alpha)}=1, c_{i}^{(\alpha)}=\left(1-\frac{1+\alpha}{i}\right) c_{i-1}^{(\alpha)} .
$$

In addition, we use the following polynomial MFs

$$
\phi_{m}(t)=t^{M+\bar{a}+1-m}(T-t)^{\bar{a}+m},
$$

where $m=1,2, \ldots, M$ with $M$ is the total number of MFs, $\bar{a}$ is an integer parameter. The fractional derivative of (39) and its 
gradient with respect to $\alpha$ can be computed analytically and are given by [14]

$D_{t}^{\alpha} \phi_{m}(t)=\sum_{k=0}^{\bar{a}+m} c_{k} \frac{\Gamma(M+\bar{a}-m+k+2)}{\Gamma(M+\bar{a}-m+k+2-\alpha)} t^{M+\bar{a}+1-m+k-\alpha}$,

where $c_{k}=\left(\begin{array}{c}\bar{a}+m \\ k\end{array}\right)(-1)^{k} T^{\bar{a}+m-k}$.

$\frac{\partial D_{t}^{\alpha} \phi_{m}(t)}{\partial \alpha}=-D_{t}^{\alpha} \phi_{m}(t)\left[\ln (t)-\psi_{0}(n-\alpha)\right], n-1<\alpha<n$.

\subsection{Estimation of parameters and fractional differentiation or-} ders

We consider the following example

$$
y(t)+a_{1} D_{t}^{\alpha_{1}} y(t)+a_{2} D_{t}^{\alpha_{2}} y(t)=u(t), t \in[0,10]
$$

where the parameters and fractional differentiation orders are $a_{1}=3, a_{2}=2$ and $\alpha_{1}=1.5, \alpha_{2}=0.5$. The input $u(t)$ is taken as a square function activated at $t=2$ and deactivated at $t=7$. The number of MFs is fixed to $M=7$.

Moreover, sensitivity analysis of the proposed algorithm to the noise level in the output and initial guess of Newton's method is performed. Table 2 shows the effect of the noise level in the output while taking the initial conditions of the fractional differentiation orders as $\alpha_{1}^{0}=1.7$ and $\alpha_{2}^{0}=0.7$. In table 3, we summarize the results of the relative errors of the estimated unknowns when the noise in the output is fixed to $5 \%$ and the initial guess is varying.

\begin{tabular}{|c|c|c|c|c|}
\hline & $\hat{\alpha}_{1}$ & $\hat{\alpha}_{2}$ & $\hat{a}_{1}$ & $\hat{a}_{2}$ \\
\hline noise-free & $4 \times 10^{-2}$ & $2 \times 10^{-2}$ & $2 \times 10^{-2}$ & $4.5 \times 10^{-2}$ \\
\hline $1 \%$ noise & 0.55 & 1.26 & 0.84 & 1.62 \\
\hline $3 \%$ noise & 1.47 & 3.04 & 1.97 & 4.04 \\
\hline $5 \%$ noise & 1.65 & 2.84 & 1.51 & 3.87 \\
\hline $10 \%$ noise & 2.9 & 5.44 & 3.33 & 7.31 \\
\hline
\end{tabular}

Table 2: Relative errors (\%) of estimated parameters and fractional differentiation orders with $\alpha_{1}^{0}=1.7$ and $\alpha_{2}^{0}=0.7$.

\begin{tabular}{|c|c|c|c|c|}
\hline & $\hat{\alpha}_{1}$ & $\hat{\alpha}_{2}$ & $\hat{a}_{1}$ & $\hat{a}_{2}$ \\
\hline$\alpha_{1,2}^{0}=1.75,0.4$ & 0.63 & 2.34 & 2.08 & 2.69 \\
\hline$\alpha_{1,2}^{0}=1.3,0.7$ & 4.23 & 7.6 & 4.94 & 10.75 \\
\hline$\alpha_{1,2}^{0}=1.3,0.3$ & 5.96 & 16.5 & 10.19 & 18.95 \\
\hline
\end{tabular}

Table 3: Relative errors (\%) of estimated parameters and fractional differentiation orders with different initial guesses of the fractional orders and output corrupted by $5 \%$ of noise

\subsection{Estimation of the input and fractional differentiation or-} ders for a fractional neurovascular model

A potential application of the proposed method is to estimate the neural activity and the fractional differentiation orders from cerebral blood flow (CBF) measurements. In [22], a fractional model for the neurovascular coupling in the brain has shown a better characterization of the cerebral hemodynamic response. This model relates the neural activity, considered as input, to the $\mathrm{CBF}$ considered as output. In order to fully identify the fractional neurovascular model, the input and the fractional differentiation orders have to be estimated from CBF data. The nature of the neural activity can be of two types: the block design paradigm which corresponds to tasks lasting for extended periods of time and the event-related paradigm which corresponds to tasks lasting for shorter periods. In this paper, we are interested in estimating the event-related type of neural activity usually modeled by Gaussian functions.

The neurovascular model is given as follows

$$
D_{t}^{\alpha_{1}} f(t)+k D_{t}^{\alpha_{2}} f(t)+\gamma f(t)=u(t), t \in[0,10],
$$

where $1 \leq \alpha_{1} \leq 2,0 \leq \alpha_{2} \leq 1$ are the fractional differentiation orders, $f(t)$ is the CBF and $u(t)$ is the neuronal activity. The constants $k$ and $\gamma$ are the flow signal decay rate and the flowdependant elimination constant, respectively. Their values are known $k=0.65, \gamma=0.41$ as given in [23]. In this work, we use a synthetic data set which have been generated from discretizing the neurovascular model using (37). The resulted signal has been perturbed by a zero mean Gaussian noise.

Before jointly estimating the input and the fractional differentiation orders of equation (43), an estimation of the unknown input is performed in noise-free and noisy cases assuming that the fractional orders are known. This step is important to show the effect of the choice of the projection basis functions depending on the smoothness of the input. Two profiles of inputs are tested, sinusoidal $u(t)=\sin \left(\frac{\pi}{T} t\right)$ and Gaussian $u(t)=\exp \left[-(t-5)^{2}\right]$.
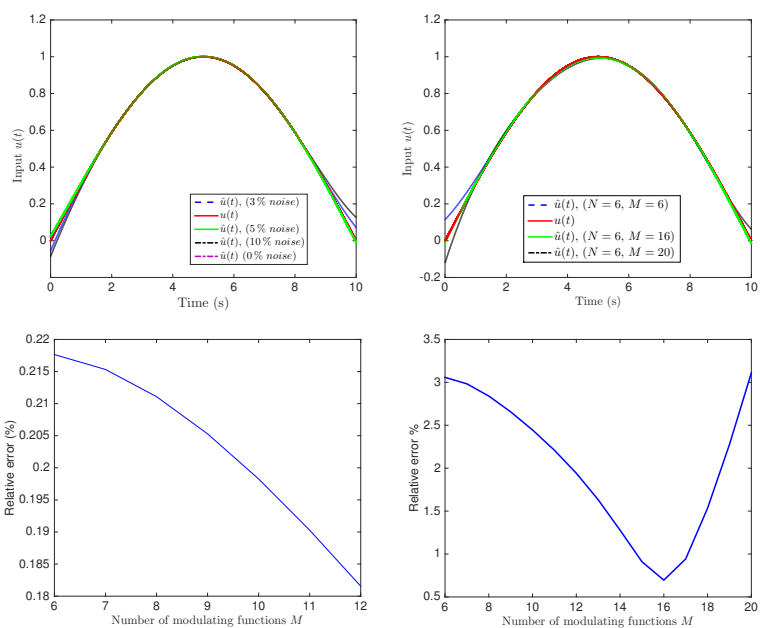

Figure 1: Estimated input for different noise levels, same number of MFs (upper left panel). Estimated input for $5 \%$ of noise with different values of $M$ (upper right panel). Relative estimation error VS number of MFs in noise-free case (lower left panel) and in noisy case (lower right panel).

Figure 1 summarizes the reconstruction results of a sinusoidal input in absence and in presence of noise using a polynomial projection basis with basis functions $\left\{t^{i-1}\right\}_{i=1}^{N}$. Following the obtained results, in noise-free case, it is sufficient to take the number of MFs equal to the number of basis functions (i.e., equal the number of unknowns). Increasing this number will not affect the performance of the algorithm significantly, as 
shown from the relative errors plotted in the lower left panel of figure 1. The top left panel shows the effect of the noise level while the number of MFs is fixed at $M=N=6$. We observe from the plots of this panel that the estimation results are affected at the edges. The top and lower right panels show the effect of $M$ for a specific level of noise $(5 \%)$. We notice from both the estimated input profiles and the values of the relative estimation error that $M=16$ is the best value for the number of MFs in the noisy case.
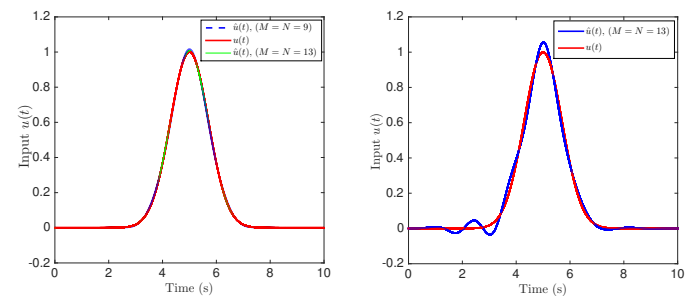

Figure 2: Estimated input in noise-free (left panel) and noisy (5\%) (right panel) cases using cubic b-splines basis with known fractional orders.

Figure 2 shows the results of estimating a Gaussian input using cubic b-splines basis functions [24] in the noise-free case and noisy case (5\% of added noise) when the fractional differentiation orders are known. We point out here that for a Gaussian input, it was not enough to use a truncated polynomial basis functions because they can not capture the main features of such type of signals.
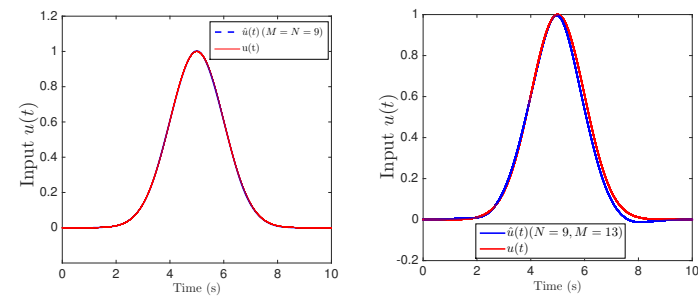

Figure 3: Estimated input in noise-free (left panel) and noisy (5\%) (right panel) cases using cubic b-splines basis with unknown fractional orders.

Figure 3 shows the results of estimating the input in noisefree case and noisy case when the fractional differentiation orders are unknown. In the noise-free case, $N=9$ and $M=N$. The final estimate of the fractional differentiation orders $\hat{\alpha}_{1}=$ 1.7 and $\hat{\alpha}_{2}=0.6$ achieve a relative input estimation error of $0.49 \%$. In the noisy case, $N=9$ and $M=N+4=13$. The final values of the fractional differentiation orders $\hat{\alpha}_{1}=1.72$ and $\hat{\alpha}_{2}=0.65$ achieve a relative input estimation error $6.35 \%$.

From the extensive numerical investigations that we conducted, the following observations are noteworthy

- The algorithm performed better in estimating the parameters compared to the input. The reason is that in addition to the numerical and noise errors contribution given in section 6, in the case of input estimation there is an expansion error due to the use of a finite set of basis functions. In this case, the finite expansion functions affect the performance of the proposed algorithm. So, the choice of the projection functions should reflect the knowledge we may have about the input (e.g. degree of smoothness, periodicity, monotonicity, etc.).

- The number $M$ of MFs affect the accuracy of the proposed algorithm. The minimal number should be equal to the number of unknown parameters $N$. In noise-free case, taking this minimum number was sufficient to give good estimates. However, in noisy case increasing the number of $M$ helped in getting more accurate results for this particular choice of MFs.

\section{Conclusion}

The estimation of the fractional differentiation orders is of great importance in fractional models describing real-life phenomena since it allows an accurate characterization of these systems. This paper has presented a hybrid approach combining the MFs algebraic method and the first-order Newton's algorithm to estimate the fractional orders jointly with the unknown parameters of non-commensurate fractional systems. An initialized definition of fractional derivative is considered in this work. Moreover, an error analysis in discrete case is proposed. Furthermore, the paper provided an extension of the proposed algorithm to the estimation of smooth unknown input simultaneously with the fractional differentiation orders. It is worth to emphasize that the proposed algorithm can be used for both commensurate and non-commensurate fractional systems. Extensive simulation results have been provided to demonstrate the performance of the proposed method.

As a future work, we will consider the estimation of a nonsmooth type of inputs, e.g., pointwise inputs modeled by a Dirac delta function. Such type of input arises in many applications such as the identification of groundwater point contaminant sources in fractional anomalous dispersion model.

\section{Appendix}

As for the uninitialized case, the proof of equation (4) follows from the Laplace transform approach. Based on [15], the Laplace transform of the fractional derivative initialized from $t=-a, a>0$ with a constant initialization function which equals to $c \in \mathbb{R}$ is given by (equation (44) of [15])

$$
\mathscr{L}\left\{D_{t}^{\alpha} f(t)\right\}=s^{\alpha} \mathscr{L}\{f(t)\}+c s^{\alpha-1}\left[\frac{e^{a s} \Gamma(1-\alpha, a s)}{\Gamma(1-\alpha)}-1\right] .
$$

Applying the convolution theorem of the Laplace transform, we get

$$
\mathscr{L}\left\{\int_{0}^{T} D_{t}^{\alpha} f(t) \phi(T-t) \mathrm{dt}\right\}=\mathscr{L}\{\phi(t)\} \mathscr{L}\left\{D_{t}^{\alpha} f(t)\right\} .
$$

Substituting the Laplace transform given in equation (44) into 
(45), we obtain

$$
\begin{aligned}
\mathscr{L} & \left\{\int_{0}^{T} D_{t}^{\alpha} f(t) \phi(T-t) \mathrm{dt}\right\}=s^{\alpha} \mathscr{L}\{f(t)\} \mathscr{L}\{\phi(t)\} \\
& +\frac{c}{\Gamma(1-\alpha)} \mathscr{L}\{\phi(t)\} \frac{e^{a s} \Gamma(1-\alpha, a s)}{s^{1-\alpha}}-c s^{\alpha-1} \mathscr{L}\{\phi(t)\} .
\end{aligned}
$$

From equation (14) of [15], the following transform applies

$\mathscr{L}\left\{(t+a)^{v}\right\}=\frac{e^{a s} \Gamma(v+1, a s)}{s^{v+1}}$, for $|\arg (a)<\pi|, \operatorname{Re}(s)>0$.

Taking into account properties (P2) and (P4) of the MFs, we can write

$$
\mathscr{L}\left\{\int_{0}^{T} D_{t}^{\alpha} \phi(t) f(T-t) \mathrm{dt}\right\}=s^{\alpha} \mathscr{L}\{\phi(t)\} \mathscr{L}\{f(t)\} .
$$

Thus, by applying the inverse of Laplace transform to (46) jointly with (47) and (48), we get

$$
\begin{gathered}
\mathscr{L}^{-1}\left\{\mathscr{L}\{\phi(t)\} \mathscr{L}\left\{D_{t}^{\alpha} f(t)\right\}\right\}(T)=\int_{0}^{T} D_{t}^{\alpha} f(t) \phi(T-t) \mathrm{dt}, \\
=\int_{0}^{T} D_{t}^{\alpha} \phi(t) f(T-t) \mathrm{dt}+\frac{c}{\Gamma(1-\alpha)} \int_{0}^{T} \phi(T-t)(t+a)^{-\alpha} \mathrm{dt} \\
-c D_{t}^{\alpha-1} \phi(T),
\end{gathered}
$$

which ends the proof.

\section{Acknowledgments}

The research work reported in this paper has been financially supported by King Abdullah University of Science and Technology (KAUST).

\section{References}

[1] A. A. Kilbas, H. M. Srivastava, J. J. Trujillo, Theory and applications of fractional differential equations, Volume 204 (North-Holland Mathematics Studies), Elsevier Science Inc., New York, NY, USA, 2006.

[2] R. Magin, Fractional calculus in bioengineering, Begell House Publisher, Inc, 2006.

[3] C. Ionescu, Emerging tools in engineering: Fractional order ladder impedance models for respiratory and neural systems, IEEE journal on emerging and selected topics in circuits and systems 3 (2013).

[4] D. Sierociuk, A. Dzielinski, Fractional kalman filter algorithm for the states parameters and order of fractional system estimation, International Journal of Applied Mathematics and Computer Science 16 (2006) 129_ 140.

[5] S. C. Lee, Y. Li, Y. Q. Chen, H. S. Ahn, $\mathrm{H}_{\infty}$ and sliding mode observers for linear time-invariant fractional-order dynamic systems with initial memory effect, Journal of Dynamic Systems, Measurement and Control 136 (2014).

[6] S. Victor, R. Malti, H. Garnier, A. Oustaloup, Parameter and differentiation order estimation in fractional models, Automatica 49 (2013) 926935.

[7] Y. Tang, H. Liu, W. Wang, Q. Lian, X. Guan, Parameter identification of fractional order systems using block pulse functions, Signal Processing 107 (2015) 272-281.

[8] R. Malti, S. Victor, A. Oustaloup, Advances in system identification using fractional models, Journal of computational and nonlinear dynamics 8 (2008).
[9] J. D. Gabano, T. Poinot, Estimation of thermal parameters using fractional modelling, Signal Processing 91 (2011) 938-948.

[10] A. Khadhraoui, K. Jelassi, J. C. Trigeassou, P. Melchior, Identification of fractional model by least-squares method and instrumental variable, Journal of computational and nonlinear dynamics 10 (2015).

[11] M. Shinbrot, On the analysis of linear and nonlinear dynamic systems from transient-response data, national advisory committee for aeronautics naca, Technical Note 3288, Washington, Tech. Rep. (1954).

[12] T. Janiczek, Generalization of modulating functions method in the fractional differential equations, Bulletin of the academy of Sciences 58 (2010) 593-599.

[13] D. Y. Liu, T. M. Laleg-Kirati, O. Gibaru, W. Perruquetti, Identification of fractional order systems using modulating functions method, in the American Control Conference, Washington, DC, USA, (2013).

[14] A. Aldoghaither, D. Y. Liu, T. M. Laleg-Kirati, Modulating functions based algorithm for the estimation of the coefficients and differentiation order for a space-fractional advection-dispersion equation, SIAM Journal on Scientific Computing (2015).

[15] C.F. Lorenzo and T.T. Hartley, Initialization of fractional-order operators and fractional differential equations, Journal of Computational and Nonlinear Dynamics 3 (2008).

[16] H. Preising, D. Rippin, Theory and application of the modulating function i. review and theory of the method and theory of the spline-type modulating functions, Computers \& Chemical Engineering 17 (1993).

[17] I. Podlubny, Fractional differential equations, New York: Academic Press, 1999

[18] C. T. Kelley, Iterative methods for linear and nonlinear equations, Society for Industrial and Applied Mathematics, SIAM, Philadelphia, 1995.

[19] K. Eriksson, D. Estep, C. Johnson, Applied mathematics: Body and Soul, Springer-Verlag Berlin Heidelberg, 2004.

[20] K. Oldham, J. Myland, J. Spanier, An atlas of functions, Springer-Verlag New York, 2009.

[21] D. Y. Liu, O. Gibaru, W. Perruquetti, T. M. Laleg-Kirati, Fractional order differentiation by integration and error analysis in noisy environment, IEEE Transactions on Automatic Control 60 (2015) 2945-2960.

[22] Z. Belkhatir, T. M. Laleg-Kirati, Fractional dynamical model for neurovascular coupling, 36th Annual International Conference of the IEEE Engineering in Medicine and Biology Society (EMBC) (2014).

[23] K. Friston, L. Harrison, W. Penny, Dynamic causal modeling, NeuroImage 19 (2003) 1273-1302.

[24] C. de Boor, A practical guide to splines, Springer-Verlag, 1978. 